\title{
edmetic
}

Revista de Educación Mediática y TIC

Influencia del contexto en el uso de dispositivos TIC en la

Formación Profesional Básica

Influence of the context on the use of ICT devices in Basic

\section{Vocational Training}

\author{
Antonio J. Moreno-Guerrero ${ }^{1}$, Jesús López Belmonte ${ }^{2}$, Santiago Pozo Sánchez ${ }^{3}$ y Arturo \\ Fuentes Cabrera ${ }^{4}$
}

Fecha de recepción: 23/08/2019; Fecha de revisión: 25/10/2019; Fecha de aceptación: $02 / 12 / 2019$

Cómo citar este artículo:

Moreno-Guerrero A.J., López, J., Pozo, S., y Fuentes, A. (2020). Influencia del contexto en el uso de dispositivos TIC en la Formación Profesional Básica. EDMETIC, Revista de Educación Mediática y TIC, 9(1), 149-169. doi: https://doi.org/10.21071/edmetic.v9i1.12195

Autor de Correspondencia: jesuslopez@ugr.es

\begin{abstract}
Resumen:
Este estudio trata de identificar en qué grado el contexto social, económico y cultural del alumnado matriculado en Formación Profesional Básica influye en el uso de dispositivos TIC, concretamente el ordenador, la Tablet y el smartphone. Se ha empleado un método cuantitativo como método de investigación y un cuestionario como instrumento para la recogida de datos, realizado por 251 alumnos de diversas familias profesionales. Se ha obtenido que estos estudiantes disponen de todo tipo de dispositivos TIC, indistintamente del contexto al que pertenecen, tienen formación en su uso y lo emplean con elevada frecuencia. Se concluye que el contexto no influye en la formación, en la frecuencia de utilización y en el uso habitual que hacen los discentes de los dispositivos TIC analizados, donde el smartphone alcanza un lugar significativo en esta población, tanto en la formación como en la frecuencia de utilización, destinándose de forma profusa para la comunicación.
\end{abstract}

Palabras clave: Condiciones culturales, Tecnología de la información, Tecnología educacional, Formación profesional, Medios electrónicos.
Abstract:
This study tries to identify to what extent the social, economic and cultural context of students enrolled in Basic Vocational Training influences the use of ICT devices, specifically the computer, the tablet and the smartphone. A quantitative method has been used as a research method and a questionnaire as an instrument for data collection, carried out by 251 students from different professional families. It has been found that these students have all kinds of ICT devices, regardless of the context to which they belong, have training in their use and use it with high frequency. It is concluded that the context does not influence the training, the frequency of use and the habitual use made by the ICT devices analyzed, where the smartphone reaches an

\footnotetext{
1 Universidad de Granada (España), ajmoreno@ugr.es;

2 Universidad de Granada (España), jesuslopez@ugr.es; (1) 0000-0003-0823-3370

3 Universidad de Granada (España), santiagopozo@correo.ugr.es; (D) 0000-0001-8125-4990

4 Universidad de Granada (España), arturofuentes@ugr.es; (D) 0000-0003-1970-4895
} 
Moreno-Guerrero, A.J., López Belmonte, J,. Pozo Sánchez, S., \& Fuentes Cabrera, A.

important place in this population, both in training and in frequency. of use, being used profusely for communication.

Key Words: Cultural conditions, Information technology, Educational technology, Vocational training, Electronic media. 


\section{INTRODUCCIÓN}

El propósito de esta investigación es describir la variable del contexto social económico y cultural del alumnado de Formación Profesional Básica, que actúa como variable independiente en la formación específica, frecuencia de uso en el ámbito personal y utilización en el ámbito escolar de dispositivos TIC, concretamente el ordenador, smartphone y Tablet.

Además, se pretende probar que el contexto socioeconómico y cultural del alumnado de Formación Profesional Básica de la Ciudad Autónoma de Ceuta influye en el uso que le dan, a nivel personal, a los dispositivos TIC anteriormente nombrados.

La variable independiente contexto socioeconómico y cultural se estructura en este estudio en tres partes claramente diferenciadas. Por un lado, el centro de la ciudad, donde el nivel que presentan los estudiantes que residen en dicha zona es medio alto; el campo exterior, donde se encuentran alumnos cuyo nivel es medio; y por último, la periferia, cuyo contexto es bajomuy bajo.

Las variables dependientes utilizadas en este estudio son formación en el uso de dispositivos TIC, que se definen como la formación que presentan a nivel personal en el manejo del ordenador, smartphone y Tablet; frecuencia de Uso de dispositivos $\mathrm{TIC}$, relacionados con el uso que hacen de manera diaria de los mismos, desde el ámbito personal; y finalmente uso de dispositivos TIC en el ámbito educativo y personal, analizando si lo usan para realizar tareas escolares, informarse, jugar, comunicarse o para otras cuestiones.

El panorama actual se instaura en una realidad en la que las tecnologías se han erigido como un elemento preponderante en nuestra vida cotidiana. La sociedad se encuentra inmersa en la era digital, de manera que no se concibe una existencia sin acceso a dispositivos tecnológicos (Del Barrio y Ruiz, 2014).

Las tecnologías de la información y la comunicación (TIC) no han pasado desapercibidas en el panorama educativo. Se han convertido en una auténtica realidad gracias a su potencialidad de uso a nivel pedagógico, originando un compendio de herramientas didácticas aprovechables tanto 
por los profesores como por el alumnado (Johnson, Adams, Gago, García y Martín, 2013; Sharples et al., 2014).

La utilización de las TIC en el contexto educativo permite ampliar las fronteras metodológicas y posibilita la creación de experiencias novedosas para los estudiantes y cercanas a su cotidianeidad (Álvarez, Delgado, Gimeno, Martín, Almaraz y Ruiz, 2017). Por su parte, Maquilón, Mirete y Avilés (2017), amplían el concepto de TIC y hacen referencia a las tecnologías para el aprendizaje y el conocimiento (TAC) para alcanzar un proceso óptimo de enseñanza-aprendizaje mediante métodos pedagógicos activos.

Actualmente, los jóvenes se encuadran en un contexto protagonizado por un continuo progreso tecnológico (Ramírez, Renés y Aguaded, 2016) que ha propiciado una inclusión integral e indudable de dispositivos electrónicos en su vida cotidiana (Castro, Caldeiro y Rodríguez, 2018). Por esta razón, varios autores han etiquetado a la actual generación de jóvenes como millennials generation (Howe y Strauss, 2007) o de nativos digitales (Prensky, 2001). Se trata de jóvenes, nacidos en el seno del mundo digital, que han vivido -con respecto a su generación anterior- un auténtico cambio de paradigma tanto en el panorama educativo como en el ámbito doméstico y del ocio.

Son varias las investigaciones que han analizado durante los últimos años las formas de uso de las TIC entre los más adolescentes (Rodríguez, Castro y Meneses, 2018), ocupando una posición privilegiada el uso del smartphone (Gaspar y Cuesta, 2017; Ramos, 2017), el ordenador (Doval, Domínguez y Álvarez, 2018; Muñoz et al., 2014) y la Tablet (Olivares, 2017; Ontano, Llanos, Pincay y Carrillo, 2018), dispositivos que la población juvenil utiliza principalmente- para la mensajería, las redes sociales y compartir información (Stald et al., 2014), tanto fuera como dentro del entorno escolar (Lenhart et al., 2015).

De esta forma, la utilización del smartphone por parte de los adolescentes constituye su sistema de comunicación prioritario (Viñals, Abad, y Aguilar, 2014) y un dispositivo tecnológico imprescindible en su vida diaria y en sus procesos de socialización (Ruíz, Sánchez y Trujillo, 2016), ya que la gran mayoría de los integrantes de la población juvenil dispone en el ámbito doméstico de un elevado número de dispositivos digitales (Melendro, García y Goig, 2016). 
Dentro del marco de la Formación Profesional Básica (en adelante, FP Básica), esta tendencia de uso frecuente de los dispositivos tecnológicos por parte de los discentes no ha pasado desapercibida. A nivel normativo, la Ley Orgánica 8/2013, de 9 de diciembre, para la mejora de la calidad educativa (LOMCE) alude a la necesidad urgente de un cambio paradigmático dentro del sistema educativo, en el que debe producirse una actualización y modernización de la Formación Profesional. Concretamente, la citada ley señala que "las TIC serán una pieza fundamental para producir el cambio metodológico que lleve a conseguir el objetivo de mejora de la calidad educativa" (p. 97864), siendo imprescindible el fomento de una utilización juiciosa basada en la responsabilidad de los discentes

Por otro lado, el Real Decreto 127/2014, de 28 de febrero, por el que se regulan aspectos específicos de la Formación Profesional Básica de las enseñanzas de formación profesional del sistema educativo, determina que los discentes deben alcanzar varias competencias relacionadas con el ámbito profesional, personal y social, entre las que destacan: "desarrollar las destrezas básicas de las fuentes de información utilizando con sentido crítico las tecnologías de la información y de la comunicación para obtener y comunicar información en el entorno personal, social o profesional" (p. 20176) y "utilizar las tecnologías de la información y de la comunicación para informarse, comunicarse, aprender y facilitarse las tareas laborales" (p. 20176).

El alumnado matriculado en esta tipología de enseñanza suele proceder de una experiencia adversa dentro de la Educación Secundaria Obligatoria (García, Pozo y Martínez, 2013; Vega y Aramendi, 2011), habiendo sufrido grandes dificultades durante la mayor parte de la etapa (González y Porto, 2013), acompañadas de bajos niveles de motivación e interés (Carrillo, 2013), de escaso rendimiento y de cuantiosas faltas de asistencia al centro de estudio (Palomares y López, 2012).

La FP Básica pretende potenciar de forma preponderante los procesos de integración del alumnado y la adaptación a sus necesidades concretas (Nogués, 2014), en base a contenidos multidisciplinares que fomenten la colaboración, la resolución autónoma de problemas y los mecanismos socializadores en su día a día y en el mundo laboral (Marco y Sancho, 2014). 
Las investigaciones realizadas por Cacheiro, García y Moreno (2016) han constatado que los alumnos de FP Básica disponen en su domicilio de ordenador y de teléfono inteligente. Sin embargo, los discentes no utilizan las TIC en el domicilio para la realización de sus tareas diarias, ya que recurren a los dispositivos tecnológicos como medio lúdico de socialización y comunicación.

A pesar de la enorme incidencia política, social y económica que las TIC tienen en el panorama actual (Seybert, 2011; Sylwester, 2003) las posibilidades de acceso varían según el lugar que ocupe el usuario potencial dentro de la escala social (Sánchez y Robles, 2016). De esta forma, se produce una evidente desigualdad de acceso a las TIC conceptualizada por varios autores como pobreza digital (Galperin y Mariscal; 2007), brecha digital (Warschaver y Ames, 2010; Warschaver, 2003) y desigualdad digital (Di Maggio, Hargittai et al, 2003).

La mencionada brecha digital tiene su origen en dos tipologías de individuo: por un lado, aquel que carece de acceso a la tecnología, y por otro, aquel que aun teniendo posibilidad de acceso, carece del conocimiento necesario para su utilización (Cabero y Ruiz, 2017).

La problemática radica en que los progresos producidos en el ámbito de la tecnología no se encuentran repartidos de forma óptima, de manera que no es recibido por todos los individuos bajo las mismas condiciones ni al mismo tiempo (Cabero, 2015) provocando grandes inequidades en el acceso a la tecnología y desigualdades en el ámbito social (Alva, 2015).

Diversas investigaciones han constatado que las clases populares tienen mayor dificultad de acceso a una computadora y a Internet con respecto a la clase media y la clase alta, existiendo un desfase de entre dos y cinco años en lo concerniente a la adquisición de esta tipología de dispositivos tecnológicos (Benítez, Monquillansky, Lemus y Welschinger, 2013; Da Silva y Ornellas, 2017).

Por todo lo expuesto, resulta primordial promover un acceso equitativo a la tecnología que permita su utilización por parte de todos los sectores de la sociedad, contribuyendo a la capacitación y formación necesaria para su utilización (Sánchez, 2008) y surtiendo de la infraestructura requerida para conseguir proveer de tecnología a todos los estratos sociales y contribuir a la eliminación de las desigualdades (Crovi, 2008; Ramírez, 2015). 


\section{MÉTODO}

La metodología aplicada en este estudio es de carácter cuantitativo y alcance descriptivo y correlacional, mediante una encuesta a 251 alumnos de Formación Profesional Básica de $1^{\circ}$ curso de la Ciudad Autónoma de Ceuta (España) cuyas características demográficas se muestran en la tabla I. La muestra aplicada es no probabilística. El diseño de investigación es de tipo no experimental, concretamente transeccional (Hernández, Fernández y Batipsta, 2014).

Tabla I. Descripción demográfica de la población. Fuente: Elaboración propia.

\begin{tabular}{lccc}
\hline \multicolumn{1}{c}{ Género } & $\mathbf{\%}$ & Edad & \% \\
\hline Masculino & 53,0 & 14 años & 1,2 \\
Femenino & 47,0 & 15 años & 21,5 \\
\hline Zona de residencia & $\%$ & 16 años & 59,0 \\
\hline Periferia de la ciudad & 45,4 & 17 años & 14,7 \\
Campo exterior & 45,0 & 18 años & 3,6 \\
Centro & 9,6 & & \\
\hline
\end{tabular}

Los datos obtenidos se analizaron a través del software SPSS. Se revisó la estadística descriptiva y correlación entre las variables demográficas, concretamente la zona de residencia (ZR), y la dimensión II del cuestionario creado ad hoc, formada por las variables formación (F) y frecuencia de uso (FS), además de la utilización habitual de los dispositivos TIC (UHD). Las pruebas aplicadas para la correlación son no paramétricas, usando la prueba de Chi Cuadrado para la asociación de variables mediante hipótesis nula. Para identificar la fuerza de relación entre variables cuya hipótesis se rechaza se ha aplicado el coeficiente Biserial por rangos, específico para asociar variables nominales y ordinales (Hernández, Fernández y Batipsta, 2014).

El instrumento utilizado es un cuestionario, al cual se le aplicó la validez de contenidos (Colás y Buendía, 1998), mediante juicio de cinco doctores expertos en la temática presentada, valorando aspectos como la claridad, adecuación e importancia de los ítems presentados. Para comprobar la fiabilidad, se aplicó la prueba a un grupo piloto de semejantes características a la población estudiada. Con los datos obtenidos se aplicó el coeficiente de consistencia interna Alfa de Cronbach (Colás y Buendía, 1998), obteniendo una valoración superior a 0,85 en cada uno de las variables analizadas, considerándose como óptima. 
El cuestionario se divide en tres dimensiones. Los datos presentados en este estudio se centran en la Dimensión II, denominada "Uso de dispositivos TIC en casa". La F analiza la formación que tiene el alumnado de FP Básica en el uso del ordenador, la tablet y el smartphone. La FS identifica la frecuencia de uso diaria de los mismos dispositivos nombrados anteriormente. Finalmente, la UHD determina, de cada uno de los dispositivos TIC, el uso que le dan para realizar trabajos de clase, informarse, jugar, comunicarse $u$ otros aspectos, tal y como se observa en la tabla Il.

Tabla II. Ítems de los instrumentos de recolección. Fuente: Elaboración propia.

\begin{tabular}{|c|c|c|}
\hline Variables & Indicadores & Ítems \\
\hline \multirow[t]{3}{*}{ Formación específica uso TIC (F) } & Formación uso ordenador & 1 \\
\hline & Formación uso tablet & 1 \\
\hline & Formación uso smartphone & 1 \\
\hline \multirow[t]{3}{*}{ Frecuencia uso TIC (FS) } & Frecuencia uso ordenador & 1 \\
\hline & Frecuencia uso tablet & 1 \\
\hline & Frecuencia uso smartphone & 1 \\
\hline \multirow{3}{*}{$\begin{array}{l}\text { Utilización habitual dispositivos TIC } \\
\text { (UHD) }\end{array}$} & Uso del ordenador en casa & 5 \\
\hline & Uso de la tablet en casa & 5 \\
\hline & $\begin{array}{l}\text { Uso del smartphone en } \\
\text { casa }\end{array}$ & 5 \\
\hline
\end{tabular}

La escala de respuesta es de tipo Likert de 4 puntos, con dos sistemas de valoraciones distintas. Para las variables F y UHD se han utilizado los puntos 1 (Nada), 2 (Poco), 3 (Bastante) y 4 (Mucho), mientras que para FS se ha aplicado una subescala de frecuencia con valoraciones que van desde el punto 1 (todos los días) hasta 4 (nunca).

La recopilación de datos se realizó en el mes de septiembre de 2014, momento en el que todos los alumnos asisten en este tipo de enseñanzas, dada su frecuente tendencia a abandonar de forma prematura los estudios en esta etapa (Moreno, 2017). En la recogida de datos participaron dos personas, formadas al respecto, desplazándose a los centros donde se impartían dichas enseñanzas (IES Abyla, IES Siete Colinas, IES Clara Campoamor, IES Almina, IES Puertas del Campo, IES Camoens y CC Severo Ochoa).

Los participantes colaboraron en todo momento y prestaron buena predisposición a la realización de la prueba, dedicando un tiempo aproximado de 30 minutos para su cumplimentación. Durante la recogida de datos, contamos con un intérprete ya que el equipo de investigación era 
consciente de las características de este tipo de alumnos, tras reuniones previas con el equipo directivo. Para evitar estos problemas, se contó en todo momento para los casos que así lo requirieran.

\section{RESULTADOS}

Los resultados que se presentan en adelante se estructuran en cuatro apartados. Los tres primeros hacen referencia al análisis descriptivo, mientras que el último al análisis correlacional.

\subsection{Zona de residencia y formación específica en el uso de dispositivos TIC.}

Tal y como se muestra en la tabla III, la formación que presenta el alumnado en el uso de dispositivos TIC es semejante, no influyendo la zona de residencia en la que se encuentren viviendo. El dispositivo TIC más destacado tiene es el smartphone, seguido de la tablet y el ordenador.

Resalta la diferencia existente en los alumnos que residen en el centro de la ciudad con el resto en la formación que tienen sobre la tablet, cuyo porcentaje duplica al resto de estudiantes residentes en otra parte de la ciudad.

Tabla III. Zona de residencia y formación específica en el uso del ordenador. Fuente: Elaboración propia.

\begin{tabular}{clllllc}
\hline $\begin{array}{c}\text { Zona } \\
\text { residencia }\end{array}$ & Nada & Poco & Bastante & Mucho & $\begin{array}{c}\text { Dispositivos } \\
\text { TIC }\end{array}$ \\
\hline Periferia de la & $\%$ & 12,3 & 17,5 & 45,6 & 24,6 & Ordenador \\
Ciudad & $\%$ & 23,7 & 14,9 & 26,3 & 35,1 & Tablet \\
& $\%$ & 19,3 & 0,9 & 8,8 & 71,1 & Smartphone \\
\hline Campo & $\%$ & $15 \%$ & $19,5 \%$ & $42,5 \%$ & $23 \%$ & Ordenador \\
Exterior & $\%$ & $27,4 \%$ & $15 \%$ & $21,2 \%$ & $36,3 \%$ & Tablet \\
& $\%$ & $17,7 \%$ & $1,8 \%$ & $13,3 \%$ & $67,3 \%$ & Smartphone \\
\hline Centro & $\%$ & $12,5 \%$ & $20,8 \%$ & $54,2 \%$ & $12,5 \%$ & Ordenador \\
ciudad & $\%$ & $16,7 \%$ & $4,2 \%$ & $12,5 \%$ & $66,7 \%$ & Tablet \\
& $\%$ & $16,7 \%$ & $0 \%$ & $4,2 \%$ & $79,2 \%$ & Smartphone \\
\hline
\end{tabular}

\subsection{Zona de residencia y frecuencia de uso de los dispositivos TIC.}

Al igual que ocurría con la formación específica en el uso de dispositivos TIC, no existe diferencia entre la frecuencia de uso de los mismos y la zona de residencia de los estudiantes, tal y como se observa en la tabla IV. El smartphone sigue siendo el dispositivo que más usan, seguido del ordenador y la tablet. Se observa una tendencia diferente a la de la formación. En este 
caso, dan más uso al ordenador que a la tablet, aun teniendo más formación específica en el uso de este dispositivo.

Como aspecto reseñable es el escaso uso dado a la Tablet por parte de los formando residentes en el centro de la ciudad.

Tabla IV. Zona de residencia y frecuencia de uso de los dispositivos TIC. Fuente: Elaboración propia.

\begin{tabular}{ccccccc}
\hline $\begin{array}{c}\text { Zona } \\
\text { residencia }\end{array}$ & $\begin{array}{c}\text { Todos los } \\
\text { días }\end{array}$ & $\begin{array}{c}\text { Dos o tres } \\
\text { veces } \\
\text { por } \\
\text { semana }\end{array}$ & $\begin{array}{c}\text { Una vez al } \\
\text { mes }\end{array}$ & Nunca & $\begin{array}{c}\text { Dispositivos } \\
\text { TIC }\end{array}$ \\
\hline Periferia de la & $\%$ & 26,3 & 36 & 11,4 & 26,3 & Ordenador \\
Ciudad & $\%$ & 13,2 & 17,5 & 19,3 & 50 & Tablet \\
& $\%$ & 86 & 1,8 & 0,9 & 11,4 & Smartphone \\
\hline Campo & $\%$ & 23,9 & 42,5 & 12,4 & 21,2 & Ordenador \\
Exterior & $\%$ & 20,4 & 23 & 11,5 & 45,1 & Tablet \\
& $\%$ & 91,2 & 2,7 & 2,7 & 3,5 & Smartphone \\
\hline Centro & $\%$ & 37,5 & 20,8 & 4,2 & 37,5 & Ordenador \\
ciudad & $\%$ & 8,3 & 54,2 & 4,2 & 33,3 & Tablet \\
& $\%$ & 91,7 & 0 & 4,2 & 4,2 & Smartphone \\
\hline
\end{tabular}

\subsection{Zona de residencia y utilización habitual de los dispositivos TIC en casa.}

En cuanto al uso habitual que le dan los estudiantes de Formación Profesional Básica a los dispositivos TIC, se observa que existen diferencias según la zona de residencia en la que se encuentre el discente, según se observa en la tabla $\vee$.

En la periferia de la ciudad y el campo exterior usan el ordenador principalmente para jugar y las tareas escolares. No lo usan para comunicarse e informarse.

En el centro de la ciudad destaca el escaso uso que le dan al ordenador para comunicarse, realizar tareas y jugar, utilizando poco dicho dispositivo para informarse.

Esta última acción es alta en todos los alumnos, dando igual la zona de residencia en la que se encuentren.

Tabla $\vee$. Zona de residencia y uso habitual del ordenador. Fuente: Elaboración propia.

\begin{tabular}{ccccccc}
\hline Zona residencia & & Nada & Poco & Bastante & Mucho & Uso \\
\hline Periferia de la & $\%$ & 44,7 & 20,2 & 19,3 & 15,8 & Tareas \\
Ciudad & $\%$ & 31,6 & 19,3 & 21,1 & 28,1 & Informarse \\
& $\%$ & 57 & 11,4 & 12,3 & 19,3 & Jugar \\
& $\%$ & 37,7 & 7 & 11,4 & 43,9 & Comunicarse \\
& $\%$ & 93,9 & 0,9 & 3,5 & 1,8 & Otras cuestiones \\
\hline Campo Exterior & $\%$ & 32,7 & 27,4 & 18,6 & 21,2 & Tareas \\
& $\%$ & 17,7 & 28,3 & 37,2 & 16,8 & Informarse \\
& $\%$ & 38,1 & 11,5 & 20,4 & 30,1 & Jugar \\
\hline
\end{tabular}




\begin{tabular}{ccccccc} 
& $\%$ & 25,7 & 11,5 & 15 & 47,8 & Comunicarse \\
& $\%$ & 86,7 & 4,4 & 6,2 & 2,7 & Otras cuestiones \\
\hline Centro ciudad & $\%$ & 45,8 & 41,7 & 0 & 12,5 & Tareas \\
& $\%$ & 20,8 & 29,2 & 29,2 & 20,8 & Informarse \\
& $\%$ & 41,7 & 16,7 & 25 & 16,7 & Jugar \\
& $\%$ & 58,3 & 4,2 & 12,5 & 25 & Comunicarse \\
& $\%$ & 100 & 0 & 0 & 0 & Otras cuestiones \\
\hline
\end{tabular}

No hay diferencias significativas en el uso habitual que hace el alumnado de FP Básica de la tablet según la zona de residencia. Donde menos lo usan es para realizar tareas escolares, informarse y comunicarse. Donde más uso le dan es para jugar, tal y como se puede observar en la tabla $\mathrm{VI}$.

Tabla VI. Zona de residencia y uso habitual de la Tablet. Fuente: Elaboración propia.

\begin{tabular}{|c|c|c|c|c|c|c|}
\hline Zona residencia & & Nada & Poco & Bastante & Mucho & Uso \\
\hline \multirow{5}{*}{$\begin{array}{l}\text { Periferia de la } \\
\text { Ciudad }\end{array}$} & $\%$ & 85,1 & 8,8 & 2,6 & 3,5 & Tareas \\
\hline & $\%$ & 78,1 & 6,1 & 7 & 8,8 & Informarse \\
\hline & $\%$ & 58,8 & 16,7 & 8,8 & 15,8 & Jugar \\
\hline & $\%$ & 61,4 & 7,9 & 10,5 & 20,2 & Comunicarse \\
\hline & $\%$ & 99,1 & 0 & 0 & 0,9 & Otras cuestiones \\
\hline \multirow{5}{*}{ Campo Exterior } & $\%$ & 82,3 & 12,4 & 5,3 & 0 & Tareas \\
\hline & $\%$ & 71,7 & 16,8 & 9,7 & 1,8 & Informarse \\
\hline & $\%$ & 43,4 & 12,4 & 15,9 & 28,3 & Jugar \\
\hline & $\%$ & 56,6 & 7,1 & 8,8 & 27,4 & Comunicarse \\
\hline & $\%$ & 91,2 & 0,9 & 3,5 & 4,4 & Otras cuestiones \\
\hline \multirow{5}{*}{ Centro ciudad } & $\%$ & 87,5 & 4,2 & 8,3 & 0 & Tareas \\
\hline & $\%$ & 58,3 & 29,2 & 12,5 & 0 & Informarse \\
\hline & $\%$ & 50 & 20,8 & 16,7 & 12,5 & Jugar \\
\hline & $\%$ & 54,2 & 20,8 & 0 & 25 & Comunicarse \\
\hline & $\%$ & 91,7 & 0 & 8,3 & 0 & Otras cuestiones \\
\hline
\end{tabular}

Finalmente, teniendo presente lo marcado en la tabla 7, los estudiantes de la periferia de la ciudad y del campo exterior utilizan el smartphone para comunicarse y jugar, mientras que los estudiantes del centro de la ciudad usan el smartphone para comunicarse e informarte. Los resultados muestran el poco uso dado de este dispositivo para la realización de tareas escolares. 
Tabla VII. Zona de residencia y uso habitual de la Smartphone. Fuente: Elaboración propia.

\begin{tabular}{ccccccc}
\hline Zona residencia & & Nada & Poco & Bastante & Mucho & Uso \\
\hline Periferia de la & $\%$ & 72,8 & 9,6 & 7 & 10,5 & Tareas \\
Ciudad & $\%$ & 36,8 & 19,3 & 21,1 & 22,8 & Informarse \\
& $\%$ & 30,7 & 9,6 & 21,1 & 38,6 & Jugar \\
& $\%$ & 14,9 & 2,6 & 1,8 & 80,7 & Comunicarse \\
& $\%$ & 87,7 & 5,3 & 2,6 & 4,4 & Otras cuestiones \\
\hline Campo Exterior & $\%$ & 69 & 11,5 & 10,6 & 8,8 & Tareas \\
& $\%$ & 35,4 & 15 & 25,7 & 23,9 & Informarse \\
& $\%$ & 22,1 & 8 & 15,9 & 54 & Jugar \\
& $\%$ & 8 & 0 & 3,5 & 88,5 & Comunicarse \\
& $\%$ & 89,4 & 0,9 & 3,5 & 6,2 & Otras cuestiones \\
\hline Centro ciudad & $\%$ & 70,8 & 16,7 & 0 & 12,5 & Tareas \\
& $\%$ & 8,3 & 37,5 & 20,8 & 33,3 & Informarse \\
& $\%$ & 25 & 25 & 25 & 25 & Jugar \\
& $\%$ & 0 & 8,3 & 4,2 & 84,9 & Comunicarse \\
& $\%$ & 95,8 & 0 & 0 & 4,2 & Otras cuestiones \\
\hline
\end{tabular}

\subsection{Correlación entre las variables formación, frecuencia de uso y uso habitual} de dispositivos con la zona de residencia.

La relación establecida entre las variables F, FS y UHD con la variable zona de residencia, con una población de 251 sujetos y un nivel de confianza del $95 \%$, muestra que existe relación entre la zona de residencia y la frecuencia de uso de la Tablet $\left(X^{2}(1 \mathrm{~N}=251)=0,003, p<.05\right)$, la utilización del ordenador para informarse $\left(X^{2}(1 \mathrm{~N}=251)=0,019, p<.05\right)$, uso de la Tablet para informarse $\left(X^{2}(1 \mathrm{~N}=251)=0,004, \quad p<.05\right), \quad$ manejo del smartphone para jugar $\left(X^{2}(1 N=251)=0,032, \quad p<.05\right) \quad$ y comunicarse $\quad\left(X^{2}(1 N=251)=0,030, \quad p<.05\right)$ rechazándose en todos estos casos la hipótesis nula, tal y como se observa en la tabla VIII. 
Tabla VIII. Correlación F, FS y UHD con zona de residencia. Prueba de Chi Cuadrado. Fuente: Elaboración propia.

\begin{tabular}{|c|c|c|c|}
\hline & Variable & Ítems & Zona de residencia \\
\hline & & Uso del ordenador & 0,878 \\
\hline & Formación & Uso de la Tablet & 0,120 \\
\hline & & Uso del smartphone & 0,766 \\
\hline & & Ordenador & 0,284 \\
\hline & vencia de uso & Tablet & 0,003 \\
\hline & & Smartphone & 0,260 \\
\hline & Ordenador & Tareas escolares & 0,058 \\
\hline E & & Informarse & 0,019 \\
\hline$\stackrel{\circ}{\geq}$ & & Jugar & 0,075 \\
\hline 跑 & & Comunicarse & 0,067 \\
\hline 0 & & Otras cuestiones & 0,319 \\
\hline$\overline{0}$ & Tablet & Tareas escolares & 0,210 \\
\hline$\overline{0}$ & & Informarse & 0,004 \\
\hline$\overline{0}$ & & Jugar & 0,070 \\
\hline$?$ & & Comunicarse & 0,202 \\
\hline$\frac{\mathrm{g}}{0}$ & & Otras cuestiones & 0,059 \\
\hline$\stackrel{c}{ᄃ}$ & Smartphone & Tareas escolares & 0,625 \\
\hline : & & Informarse & 0,067 \\
\hline O & & Jugar & 0,032 \\
\hline 谓 & & Comunicarse & 0,030 \\
\hline & & Otras cuestiones & 0,411 \\
\hline
\end{tabular}

Con respecto a la fuerza de asociación, los valores que se obtienen tras aplicar el coeficiente biserial por rangos entre la zona de residencia y la frecuencia de uso de la Tablet $(-0,129)$, la utilización del ordenador para informarse (0.025), uso de la Tablet para informarse (0.004), manejo del smartphone para jugar (0.040) y comunicarse (0.129), se observa que no hay fuerza de relación entre las variables, dada que la correlación en unos casos es débil o muy débil, no pudiéndose demostrar la relación entre las variables.

\section{4, DISCUSIÓN/CONCLUSIONES}

El alumnado de FP Básica dispone de todos los dispositivos posibles, tal y como establecen Del Barrio y Ruiz (2014), los cuales determinan que las tecnologías se encuentran de lleno en nuestra vida diaria, aun procediendo la mayoría de discentes un contexto social, económico y cultural medio-bajo, estando en contraposición a lo indicado por autores como Galperin y Mariscal (2007) o Wrschaver y Ames (2010). El contexto no influye en la formación que tiene el alumno en relación a los dispositivos TIC, la cual es alta. 
Los alumnos muestran mayor grado de formación en el smartphone, seguido de la Tablet y el ordenador, estando en consonancia con lo marcado por Howe y Strauss (2007) o Prensky (2001), los cuales consideran a esta generación de jóvenes como una generación digital.

Los estudiantes de FP Básica que residen en el centro de la ciudad tienen una formación más elevada en el uso de la Tablet que el resto de estudiantes de otras zonas.

El contexto no influye en la frecuencia de uso de los dispositivos TIC, algo en contra a lo establecido por Benítez, Monquillansky, Lemus y Welschinger (2013) o Da Silva y Ornellas (2017).

El dispositivo TIC con más frecuencia de uso es el smartphone, tal y como demuestran los estudios realizados por Gaspar y Cuesta (2017), seguido del ordenador, según establecen Doval, Domínguez y Álvarez (2918) y la Tablet, verificado por Olivares (2017). Sin embargo, esta encadenación de recursos electrónicos no se produce de forma análoga en el ámbito formativo, existiendo discrepancias entre la frecuencia de uso de los mismos.

Los estudiantes residentes en el centro de la ciudad, aun teniendo formación en el uso de la Tablet, se ha obtenido que es el dispositivo que utilizan con menor frecuencia. En cambio, los discentes que residen en la periferia y campo exterior de la ciudad ni demuestran un uso frecuente ni una formación especializada en dicho dispositivo.

En relación al uso específico que hace el alumnado de FP Básica con respecto a cada uno de los dispositivos TIC analizados, se encuentra que los participantes residentes en la periferia y campo exterior de la ciudad emplean el ordenador - principalmente- para comunicarse e informarse, pero no acuden a tal dispositivo para jugar o realizar tareas escolares, en contraposición con lo marcado por Johnson et al. (2013), quienes consideran que estos dispositivos generan un alto potencial didáctico.

Por otro lado, los alumnos residentes en el centro de la ciudad utilizan el ordenador como herramienta comunicativa, lúdica y para la realización de tareas escolares, pero no para un uso informativo.

En relación al uso de la Tablet, a pesar de su baja frecuencia de utilización, no hay diferencias según la zona de residencia del estudiante, 
siendo su uso mayoritario de índole lúdica y utilizándola con escasa frecuencia para comunicarse, informarse o realizar tareas escolares.

Con respecto al smartphone, los estudiantes lo emplean con una alta frecuencia, destinando su uso - fundamentalmente- para comunicarse, y en menor grado para la realización de tareas escolares, de acuerdo con Viñals, Abad y Aguilar (2014), resultando indiferente la zona de residencia.

En consonancia con Stald et al. (2014), se obtiene que el uso mayoritario de los distintos dispositivos estudiados es para fines comunicativos; sin embargo, estos se emplean minoritariamente para la realización de tareas escolares, tal y como establecen Cacheiro, García y Moreno (2016).

Podemos decir a modo de conclusión que el alumnado de FP Básica de la Ciudad Autónoma de Ceuta dispone de ordenador, smartphone o Tablet, con independencia de la zona de residencia. Este hecho revela unos resultados contrarios a estudios previos en los que se analizó el uso y la formación de las TIC en un contexto social, económico y cultural medio-bajo.

Desde el punto de vista discente, la formación que presentan en los dispositivos TIC analizados es alta, estando estas destrezas digitales enfocadas hacia el ocio, específicamente el aspecto lúdico y comunicativo.

El dispositivo $\mathrm{TIC}$ en el que los alumnos presentan mayor competencia digital es el smartphone, con independencia de su zona de residencia.

En función de la zona de residencia de los estudiantes, los dispositivos en los que presentan mayor destreza en su uso son el ordenador y la Tablet, siendo esta última la más utilizada por los discentes que viven en el centro de la ciudad, y el ordenador el dispositivo con mayor frecuencia de uso en el campo exterior.

Con respecto a la frecuencia de uso, independientemente de la zona de residencia, es el smartphone el dispositivo más utilizado, seguido del ordenador y la Tablet.

La principal utilización de los distintos dispositivos efectuada por los estudiantes corresponde con el ámbito comunicativo y lúdico, ocupando el smartphone un lugar preponderante para tal fin.

En base a los resultados obtenidos, no se puede afirmar que exista correlación entre el contexto del alumnado de FP Básica y la formación, 
frecuencia de utilización y uso habitual de los dispositivos, dado que las correlaciones que se establecen presentan escasa fuerza de asociación.

Se concluye que el contexto no influye en la formación, frecuencia de utilización y uso habitual que hacen los estudiantes de los dispositivos TIC analizados, donde el smartphone alcanza un lugar significativo en esta población, tanto en la formación como en la frecuencia de utilización, destinándose de forma profusa para la comunicación.

De cara a futuras investigaciones, se pretende analizar el motivo por el cual el uso de dispositivos TIC dentro de esta población de estudio está más orientado al ocio que a la formación académica. Asimismo se ampliará la muestra a etapas superiores, tales como Ciclos Formativos de Grado Medio y Ciclos Formativos de Grado Superior.

\section{REFERENCIAS}

ALVA, A. R. (2015). Los nuevos rostros de la desigualdad en el siglo XXI: la brecha digital. Revista Mexicana de Ciencias Políticas y Sociales, $60(223), 265-285$.

Álvarez, S., delGAdO, L., GIMENO, M. A., MARTín, T., AlMARAZ, F., y RUIZ, C. (2017). El arenero educativo: la realidad aumentada un nuevo recurso para la enseñanza. EDMETIC, 6(1), 105-123. doi. https://doi.org/10.21071/edmetic.v6i1.5810

BENÍTEZ, S., MOGUILLANSKY, M., LEMUS, M., y WELSCHINGER, N. (2013). TIC, clase social y género: La constitución de desigualdades sociales y digitales en las juventudes argentinas. En E. Crenzel (Presidencia), X Jornadas de Sociología 1 al 6 de julio de 2013 Ciudad Autónoma de Buenos Aires. 20 años de pensar y repensar la sociología. Nuevos desafíos académicos, científicos y políticos para el siglo XXI. Facultad de Ciencias Sociales, Buenos Aires.

CABERO, J. (2015). Reflexiones educativas sobre las tecnologías de la información y la comunicación (TIC). Tecnología, Ciencia y Educación, $1,19-27$.

CABERO, J., y RUIZ, J. (2017). Las Tecnologías de la Información y Comunicación para la inclusión: reformulando la brecha digital. IJERI. 
International Journal of Educational Research and Innovation, 9, 16-30. Google Scholar

CACHEIRO, M. L., GARCÍA, F., Y MORENO, A. J. (2016). Las TIC en los programas de Formación Profesional Básica en Ceuta. Apertura, 7(2), 132-151. Google Scholar

CARRILLO, O. (2013). Comprendiendo la adquisición de las competencias ciudadanas en alumnos de los programas de cualificación profesional inicial. Educar, 49(2), 207-226.

CASTRO, A., CALDEIRO, M. C., y RODRÍGUEZ, M. M. (2018). El uso de smartphones y tablets en Educación Infantil. Una propuesta de investigación que empodera a la infancia. Aula Abierta, 47(3), 273-280. doi. 10.17811/rifie.47.3.2018.273-280 Google Scholar

COLÁS, M.P., y BUENDÍA, L. (1998). Investigación Educativa. 3. a Edición. Sevilla: Ediciones Alfar.

CROVI, D. (2008). Dimensión social del acceso, uso y apropiación de las TIC. Contratexto, (16), 65-79, doi: 10.26439/contratexto2008.n016.784 Google Scholar

DA SILVA, M., y ORNELLAS, A. (2017). Potencialidades de un modelo colaborativo para la apropiación de las TIC en un contexto de vulnerabilidad social. Revista Psicopedagogia, 34(104), 216-227. Google Scholar

DEL BARRIO, Á., y RUIZ, I. (2014). Los adolescentes y el uso de las redes sociales. International Journal of Developmental and Educational Psychology (Revista INFAD de Psicología), 3(1), 571-576 doi: https://doi.org/10.17060/ijodaep.2014.n1.v3.537. Google Scholar

DI MAGGIO, P., HARGITTAI, E., NEUMAN, W. R., y ROBINSON, J. P. (2003). Social implications of the Internet. Annual Review of Sociology, 27(1), 307-336. doi: https://doi.org/10.1146/annurev.soc.27.1.307

DOVAL, M., DOMÍNGUEZ, S., y ÁLVAREZ, I. D. (2018). El uso ritual de las pantallas entre jóvenes universitarios/as. Una experiencia de dieta digital. Prisma Social: Revista de Investigación Social, 21, 480-499. 
GALPERIN, H., y MARISCAL, J. (2007). Poverty and mobile telephony in Latin America and the Caribbean. Dialogo Regional sobre Sociedad de la Información. Otawa, Canadá: DIRSIIIDRC.

GARCÍA, B., POZO, M., y MARTíNEZ, M. (2013). Los programas de cualificación profesional inicial. Un estudio de casos. XVI Congreso Nacional/II Internacional Modelos de Investigación Educativa. Recuperado de http://dialnet.unirioja.es/servlet/articulo? codigo $=4377268$

GASPAR, S., y CUESTA, V. (2016). El uso del smartphone entre jóvenes: un análisis comparativo cross-cultural. Nuevas Formas de Expresión en Comunicación, 8, 359-374.

GONZÁlEZ, M., y PORTO, M. (2013). Programas de Cualificación Profesional Inicial: valoraciones e implicación de los alumnos en la Comunidad Autónoma de Murcia. Revista de Educación, extraodrinaio 2013, 210235. Recuperado de http://www.mecd.gob.es/dctm/revista-deeducacion/articulosre2013

HERNÁNDEZ, R., FERNÁNDEZ, C., y BATIPSTA, M.P. (2014). Metodología de la investigación. México D.F.: McGraw-Hill Education.

HOWE, N., y STRAUSS, W. (2007). The next 20 years. Harvard Business Review, $85(7-8), 41-52$.

JOHNSON, L., ADAMS, S., GAGO, D., GARCÍA, E., y MARTíN, S. (2013). Technological Perspectives: Higher Education in Latin America 20132018. A regional analysis of the Horizon report, Texas, EEUU: The New Media Consortium.

LENHART, A., DUGGAN, M., PERRIN, A., STEPLER, R., RAINIE, H., y PARKER, K. (2015). Teens, social media \& technology overview, Sydney, Australia: Pew Research Center.

Ley Orgánica 8/2013, de 9 de diciembre, para la mejora de la calidad educativa. «BOE» núm. 295, Sec. I. Pág. 97858-97921.

MAQUILÓN, J.J., MIRETE, A.B., y AVILÉS, M. (2017). La Realidad Aumentada (RA). Recursos y propuestas para la innovación educativa. Revista Electrónica Interuniversitaria de Formación del Profesorado, 20(2), 183-204. doi. https://doi.org/10.6018/reifop/20.2.290971. 
MELENDRO, M., GARCíA, F. J., y GOIG, R. (2016). El uso de las TIC en el ocio y la formación de los jóvenes vulnerables. Revista Española de Pedagogía, (263). 71-89.

MORENO, A. J. (2017). Perfil del alumnado de Formación Profesional Básica de la Ciudad Autónoma de Ceuta. En JM Alcántara, M. Bermúdez, FJ: Blanco y JM Heredia (Ed.), Investigación e innovación en el ámbito universitario. Tendencias ante los retos actuales de la sociedad (pp.283296). Madrid: Editorial EOS Universitaria.

MUÑOZ, R., ORTEGA, R., BATALLA, C., LÓPEZ, M. R., MANRESA, J. M., Y TORÁN, P. (2014). Acceso y uso de nuevas tecnologías entre los jóvenes de educación secundaria, implicaciones en salud. Estudio JOITIC. Atención Primaria, 46(2), 77-88. doi. https://doi.org/10.1016/j.aprim.2013.06.001 Google Scholar

OLIVARES, M. Á. (2017). La adicción y la violencia en el tiempo libre de niños y jóvenes desde el teléfono, la tablet o el videojuego. Misión joven: revista de pastoral juvenil, (485), 5-18. Google Scholar

ONTANO, M. A., LLANOS, G. L., PINCAY, S. D., y CARRILLO, P. S. C. (2018). Eficiencia de las Tablets en la Educación. RECIAMUC, $2(2), 247-255$. Google Schọlar

PALOMARES, A., y LÓPEZ, S. (2012). La respuesta a la diversidad: de los programas de garantía social hacia los programas de cualificación profesional inicial. Revista Española de Educación Comparada, 20, 275302. doi: 10.5977/reec.20.2012.76300

PRENSKY, M. (2001). Digital natives, digital immigrants part 1. On the horizon, $9(5), 1-6$.

RAMÍREZ M. S. (2015). Acceso abierto y su repercusión en la Sociedad del Conocimiento: Reflexiones de casos prácticos en Latinoamérica. Education in the Knowledge Society, 16(1), 103-118. doi. http://dx.doi.org/10.14201/eks2015161103118

RAMÍREZ, A., RENÉS, P., y AGUADED, I. (2016). La competencia mediática en los criterios de evaluación del currículo de Educación Primaria. Aula 
Abierta, 44(2), 55-62. doi: https://doi.org/10.1016/j.aula.2015.08.002 Google Scholar

RAMOS, R. (2017). Smartphones as an extension of the human cyborg: the case of the youth from Aragon (Spain). Anàlisi, 56, 101-115.

Real Decreto 127/2014, de 28 de febrero, por el que se regulan aspectos específicos de la Formación Profesional Básica de las enseñanzas de formación profesional del sistema educativo, BOE Núm. 55; Sec. I. Pág. 20155- 21136.

RODRÍGUEZ, D., CASTRO, D., y MENESES, J. (2018). Usos problemáticos de las TIC entre jóvenes en su vida personal y escolar. Comunicar, 56, 91-100. doi: https://doi.org/10.3916/C56-2018-09

RUIZ, J., SÁNCHEZ, J., y TRUJILLO, J. M. (2016). Utilización de Internet y dependencia a teléfonos móviles en adolescentes. Revista Latinoamericana de Ciencias Sociales, Niñez y Juventud, 14(2), 13571369.

SÁNCHEZ, D., y ROBLES, M. A. (2016). Riesgos y potencialidades de la era digital para la infancia y la adolescencia. Educación y Humanismo, 18(31), 186204.

SÁNCHEZ, E. (2008). Las tecnologías de información y comunicación (TIC) desde una perspectiva social. Revista Electrónica Educare, 12, 155-162. Google Scholar

SEYBERT, H. (2011). Internet use in households and by individuals in 2011 . Eurostat statistics in focus, 66, 1-8.

SHARPLES, M., ADAMS, A., FERGUSON, R., GAVED, M., MCANDREW, P., RIENTIES, B., et al. (2014). Innovating pedagogy 2014. Milton Keynes, United Kingdom: Open University.

STALD, G., GREEN, L., BARBOVSKI, M., HADDON, L., MASCHERONI, G., SÁGVÁRI, B., et al. (2014). Online on the mobile: Internet use on smartphones and associated risks among youth in Europe, Perth, Australia: Edith Cowan University.

SYLWESTER, R. (2003). A biological brain in a cultural classroom: Enhancing cognitive and social development through collaborative classroom management. Thousand Oaks, CA: Corwin Press. 
VEGA, A., y ARAMENDI, P. (2010). Entre el fracaso y la esperanza. Necesidades formativas del alumnado de los Programas de Cualificación Profesional Inicial. Educación XX1, 13(1), 39-63. Recuperado de http://revistas.uned.es/index.php/educacionXX1/article/view/276 Google Scholar

VIÑALS, A., ABAD, M., y AGUILAR, E. (2014). Jóvenes conectados: una aproximación al ocio digital de los jóvenes españoles. Communication papers, 3(4), 52-68.

WARSCHAUER, M. (2003) Social capital and access. Universal access in the Information Society, 2(4), 15-31.

WARSCHAUER, M., y AMES, M. (2010). Can One Laptop per Child save the world's poor? Journal of International Affairs, 33-51. 